\title{
Mass Sensitivity Evaluation of Surface Acoustic Wave Biosensor
}

\author{
Chen Chen ${ }^{1, *}$, and Yuntao Xie ${ }^{1}$ \\ ${ }^{1}$ Changchun University of Science and Technology, No. 7089 Weixing Road, Changchun 130022, China
}

\begin{abstract}
In this paper, the mass sensitivity of a 3-Dimensional $\mathrm{SiO}_{2} / 36^{\circ} \mathrm{YX} \mathrm{LiTaO}_{3}$ Love mode SAW biosensor is investigated by a novel approach. The normalized displacement confirms the shear horizontal nature of Love wave. The phase mass sensitivity of $76.4931 \mathrm{~m}^{2} / \mathrm{kg}$ is obtained by evaluating the phase shifts. The insertion loss of $-17.2 \mathrm{~dB}$ is achieved by impulse response analysis. This Love mode SAW biosensor has potential applications in detecting viruses, cancer markers, bacteria, etc.
\end{abstract}

\section{Introduction}

Biosensors [1] are analytical devices used for the detection of analytes. They combine biologically sensitive elements called bioreceptors with physicochemical transducers. These bioreceptors are biological materials that can directly bind to target analytes in a sample mixture including tissues, cells, enzymes, antibodies, and nucleic acids. Based on the type of transducers, biosensors can be classified as electrochemical, optical, gravimetric, and piezoelectric biosensors [2-4].

Surface acoustic wave (SAW) piezoelectric biosensors are one of the most promising sensing platforms because they are highly sensitive to surface perturbations, can operate in both dry and aqueous environments, have wireless operation capability and can be easily fabrication by standard microfabrication techniques.

Love (or SH) mode SAW with the dominant displacement parallel to the substrate surface provides a low damping in liquid [5-6]. Love SAW can be generated in a layered structure consisting of a substrate and a waveguide layer when the acoustic velocity of the waveguide layer is lower than that of the substrate. Due to the waveguide effect, most of the wave energy is confined to the waveguide layer. Therefore, Love wave device is very sensitive to the surface perturbation and is suitable for sensor applications especially in liquid [6-7]. However, with the growing need for extremely high sensitive devices, maximizing sensitivity of such sensors is of utmost importance.

In this paper, the sensitivity of Love wave sensor is investigated by a novel approach. The time delays due to the thickness changes of the sensing film are measured at the output IDT. The phase shifts associated with delays are used to determine the phase sensitivity.

\section{Simulation Method}

The sensitivity is the quantitative relation between the variations of the acoustic signal and the physical modifications occurring in the environment above the sensing area. The phase mass sensitivity $\left(\mathrm{S}_{\mathrm{m}^{\phi}}\right)$ for SAW devices in delay line relates the surface density change to the electrical phase change as stated below [8]:

$$
S_{m \varphi}=\lim _{\Delta \mathrm{m} \rightarrow 0} \frac{1}{k D} \frac{\Delta \varphi}{\Delta \mathrm{m}} .
$$

where $\Delta \varphi$ denotes the phase shift occurring as a result of application of incremental mass per unit area $\Delta \mathrm{m}$ over the mass loading region, $\mathrm{D}$ is the distance between input and output IDT and $\mathrm{k}$ is the wavevector.

\subsection{Mathematical Method}

The piezoelectric constitutive equations used are

$$
\begin{aligned}
T_{i j}= & c_{i j}{ }_{l} S \quad{ }_{l}-e{ }_{i j} E_{k} \\
& D_{i}=e_{i j} \quad{ }^{j}+\varepsilon_{i j} E_{j}
\end{aligned}
$$

(2) which couple the mechanical effect as governed by Newton's law and the electrical effect as determined by Gauss law, see reference[9]. Where $T_{i j}$ is the stress matrix, $\mathrm{S}_{\mathrm{jk}}$ is the strain matrix, Di is the electric displacement vector, $E_{j}$ is the applied electric field vector and subscripts $\mathrm{i}, \mathrm{j}, \mathrm{k}, \mathrm{l}=\mathrm{x}, \mathrm{y}, \mathrm{z}$. The material constants used as input are the elasticity (mechanical stiffness) matrix $\mathrm{c}_{\mathrm{ijkl}}$, the piezoelectric coupling matrix $e_{\mathrm{ijk}}$ and the permittivity matrix $\varepsilon_{\mathrm{ij}}$.

\subsection{FEM Model}

FEM is used to solve the above equations in 3-dimensions. The device design and geometry are shown in Fig. 1. The two-port delay line structure consists of two finger pairs in each port. Massless IDT electrodes is considered to avoid the mass loading effect on the 
substrate. The device operates at $\lambda=12 \mu \mathrm{m}$. Model parameters are displayed in Table 1. Since piezoelectric crystals are anisotropic, a proper crystal cut and wave propagation direction is essential for simulation. The rotated coordinate system is used to rotate the $\mathrm{LiTaO}_{3}$ crystal to get the desired orientation of $36^{\circ} \mathrm{YX} \mathrm{LiTaO}_{3}$. The properties of materials used in simulation are from references [10] and [11].

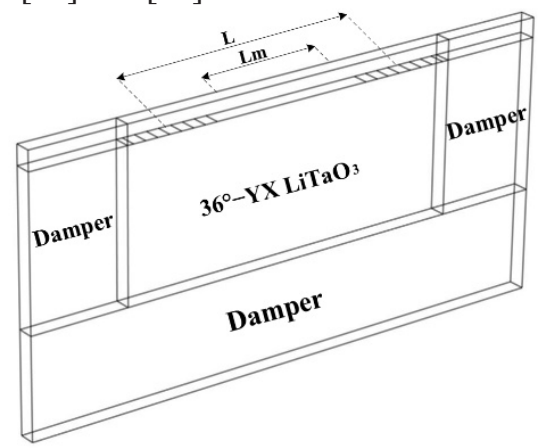

Fig. 1. 3D simulation model of the Love mode SAW delay line.

Table 1. Model dimensions and geometry.

\begin{tabular}{lc}
\hline \multicolumn{1}{c}{ Geometry } & Value \\
\hline IDT center to center distance (L) & $5 \lambda$ \\
Sensitive area length $(\mathrm{Lm})$ & $3 \lambda$ \\
IDT finger width & $\lambda / 4$ \\
IDT finger length & $1 \mu \mathrm{m}$ \\
Substrate length & $1 \mu \mathrm{m}$ \\
Substrate thickness & $3 \lambda$ \\
\hline
\end{tabular}

\section{Results and Discussion}

\subsection{Harmonic Response and Mass Sensitivity Analysis}

A sinusoidal voltage of $\mathrm{V}_{\text {in }}=5 \times \sin (2 \pi \mathrm{ft}) \mathrm{V}$ is applied to the input IDT. The total simulation time is run for $40 \mathrm{~ns}$ with a time step of $0.01 \mathrm{~ns}$. The particle displacement and voltage at the output IDT are recorded. Fig. 2 shows the displacement field at $t=8.5$ and 17.1 ns. The shearhorizontal nature of the Love wave is evident from the time response of the delay line. Fig. 3 shows input and output voltages on IDT. Input signal is harmonic from start of simulation. Output IDT needs some time to receive Love wave, and after $28 \mathrm{~ns}$ output voltage stabilizes. Application of $5 \mathrm{~V}$ sinusoidal input gives a stable $0.77 \mathrm{~V}$ sinusoidal output at the receiving IDT.

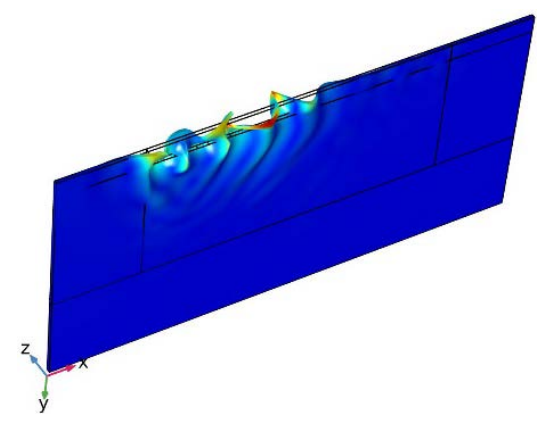

(a)

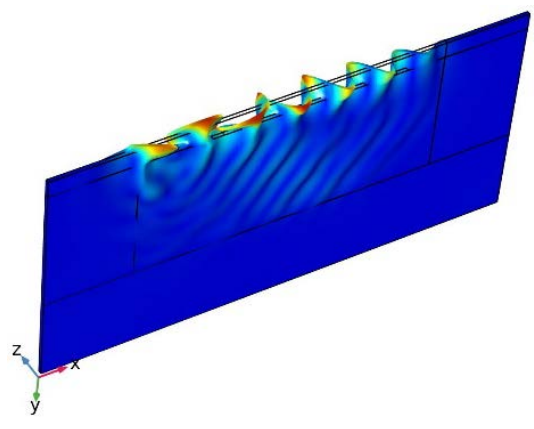

(b)

Fig. 2. Time response of Love mode SAW delay line at (a) $8.5 \mathrm{~ns}$ and (b) $17.1 \mathrm{~ns}$.

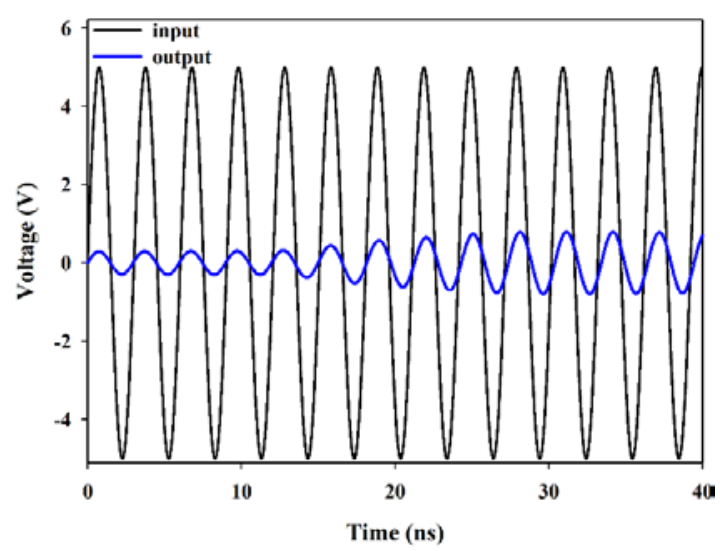

Fig. 3. Variation of input and output voltages with time.

To calculate the mass sensitivity of the Love wave delay line, a layer of $200 \mathrm{~nm}$ thick PMMA layer is placed on top of the delay area to simulate mass loading. The output voltage with and without mass loading is shown in Fig. 4. The presence of PMMA layer causes a time delay. To determine the $S_{m \phi}$, the responses of the output voltages are analyzed for five simulations in which the thickness of PMMA is increased from $180 \mathrm{~nm}$ to $340 \mathrm{~nm}$ in steps of $40 \mathrm{~nm}$. The corresponding time delay of these simulations is measured, and compared with the output voltage of the reference PMMA layer $(\mathrm{h}=180 \mathrm{~nm})$. The corresponding values of time delay and phase shift are listed in Table 2. The first row in it refers to the reference simulation. Fig. 5 shows the relation of phase shift with surface density. The slope of the curve obtained with linear regression $(y=76.4931 \mathrm{x}-0.0168)$ represents the phase mass sensitivity $\mathrm{S}_{\mathrm{m}}$, which is of $76.4931 \mathrm{~m}^{2} / \mathrm{kg}$.

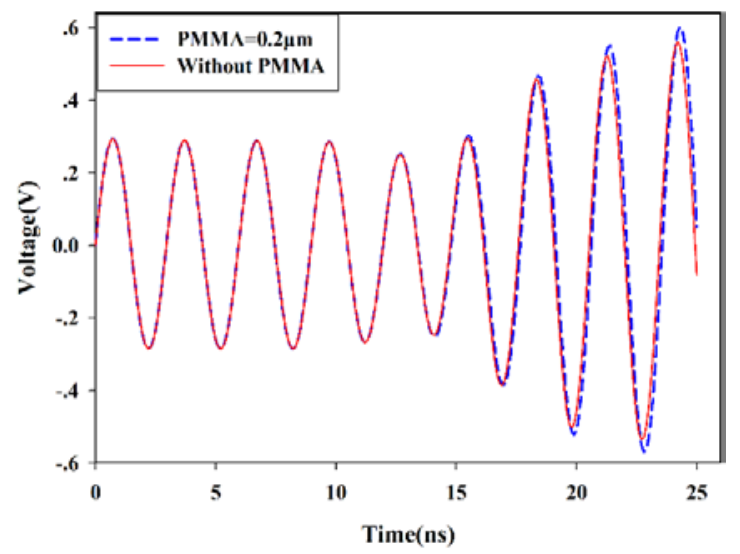

Fig. 4. Voltage at the output IDT in the absence and presence of PMMA. 
Table 2. Time delays and phase shifts for PMMA thickness variations.

\begin{tabular}{cccc}
\hline $\begin{array}{c}\text { PMMA } \\
\text { thickness }(\mathrm{nm})\end{array}$ & $\begin{array}{c}\Delta \mathrm{m} \\
\left(\mathrm{kg} / \mathrm{m}^{2}\right)\end{array}$ & $\begin{array}{c}\text { Delay } \\
\text { time }(\mathrm{ns})\end{array}$ & $\Delta \varphi(\mathrm{rad})$ \\
\hline 180 & 0.0002124 & 0 & 0 \\
220 & 0.0002596 & 0.027 & 0.056204053 \\
260 & 0.0003068 & 0.06 & 0.124897896 \\
300 & 0.000354 & 0.097 & 0.201918265 \\
340 & 0.0004012 & 0.142 & 0.295591687 \\
\hline
\end{tabular}

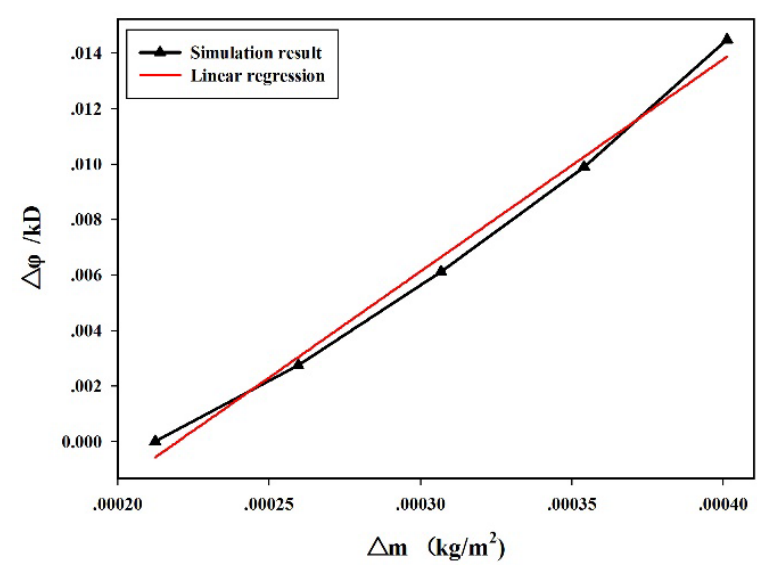

Fig. 5. Normalized phase shift $(\Delta \phi / \mathrm{kD})$ versus incremental mass per unit area $(\Delta \mathrm{m})$.

\subsection{Impulse Response and Insertion Loss Analysis}

Impulse response of the device is performed by applying a unit impulse of $1.5 \mathrm{~ns}$ width at the input IDT. The impulse response is measured at the output IDT and shown in Fig.6 (a). The insertion loss (IL) of the device is found by taking the Fourier transform of the impulse response, and calculated by the relation

$$
\operatorname{IL}(\mathrm{dB})=20 \lg \left(\left|\frac{V_{\text {out }}}{V_{\text {in }}}\right|\right) \text {. }
$$

Fig.6 (b) shows the insertion loss of the device over a frequency span of $500 \mathrm{MHz}$. The device exhibits an IL value of $-17.2 \mathrm{~dB}$ at $345 \mathrm{MHz}$. As we move away from this frequency, the losses increase considerably.

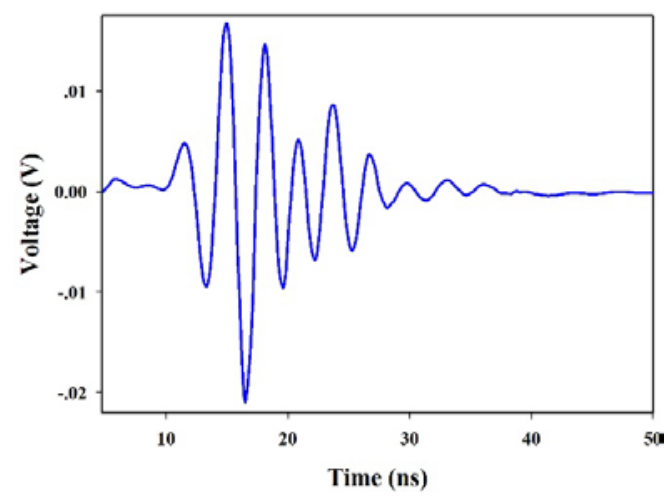

(a)

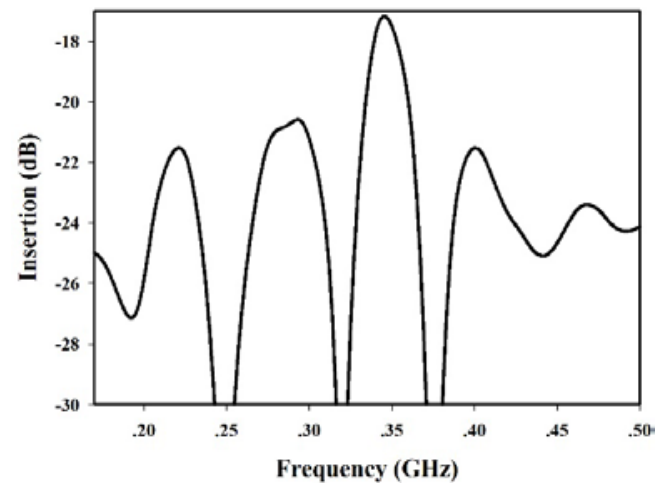

(b)

Fig. 6. (a) Impulse response (b) Insertion loss.

\section{Conclusions}

The mass sensitivity of a 3 -Dimensional $\mathrm{SiO}_{2} / 36^{\circ} \mathrm{YX}$ $\mathrm{LiTaO}_{3}$ Love mode SAW sensor with varying sensing film thickness is investigated. The calculated normalized displacement confirms the shear horizontal nature of Love wave. The output voltages of the IDT are used to acquire the time delays, which associate with phase shifts. The phase mass sensitivity of $76.4931 \mathrm{~m}^{2} / \mathrm{kg}$ and IL of $17.2 \mathrm{~dB}$ are evaluated. The results have done a great favor for optimizing of the Love mode SAW device which could be used for the biosensors, and help to obtain the device characteristics prior to actual fabrication.

The authors gratefully acknowledge the financial support of National Natural Science Foundation of China (Grant No.11604025) and Program of Science and Technology Development Plan of Jilin Province of China (Grant No.20200201249JC).

\section{References}

1. P.J. Jandas, J.T. Luo, A.J. Quan, Appl. Surface. Sci 518, 1 (2020)

2. Y.P. Fan, X.J. Ji, Acoust. Phy 64, 122 (2018)

3. Q.B. Tang, Y.J. Guo, Journ. Mater. Sci 54, 11925 (2019)

4. A.P.F. Turner, Chem. Soc. Rev 42, 3184 (2013)

5. J.T. Luo, A.J. Quan, C. Fu, Journ. Alloy. Compd 693, 558 (2017)

6. J.T. Luo, A.J. Quan, G.X. Liang, Ultrason 75, 63 (2017)

7. F. Moreira, M.E. Hakiki, F. Sarry, IEEE. Sensor 7, 336 (2007)

8. S. Houri, U. Bhaskar, L.A. Francis, Exper. Mechan 53, 441 (2013)

9. B. Auld, Acoustic Fields and Waves in Solids (John Wiley and Sons, Hobaken, 1973)

10. M. Gaso, Y. Jiménez, L. Francis, Love Wave Biosensors: A Review (IntechOpen, London, 2013)

11. G. Kovacs, M. Anhorn, H.E. Engan, Improved material constants for $\mathrm{LiNbO}_{3}$ and $\mathrm{LiTaO}_{3}$ (IEEE, Honolulu, 1990 